\title{
Design and Development of a Slender Dual-Structure Continuum Robot for In-situ Aeroengine Repair
}

\author{
Mingfeng Wang ${ }^{1}$, David Palmer ${ }^{1}$, Xin Dong $^{1 *}$, David Alatorre ${ }^{1}$, Dragos Axinte ${ }^{1}$, Andy Norton ${ }^{2}$
}

\begin{abstract}
In-situ aeroengine maintenance works (e.g. inspection, repair) are highly beneficial as it can significantly reduce currently accepted maintenance cycle which is extensive and costly due to the need to remove engines from the wing of an aircraft. However, feeding in/out via inspection ports and performing a multi-axis movement of an end-effector in a very constrained environment such as aeroengine combustion chamber is a fairly challenging task. This paper presents the design and development of a highly slender (i.e., low diameterto-length ratio) dual-structure continuum robot with 16 degrees of freedom (DoFs) to provide the feeding motion needed to navigate into confined environments and then perform a required configuration shape for further repair operation. This continuum robot is a compact system and presents a set of innovative mechatronic solutions such as: (i) two-stage tendondriven structure with bevelled disk design to perform required configuration shape and to provide selective stiffness for the ability of taking high payloads; (ii) various compliant joints to enable different flexibility requirement in each stage; (iii) three commanding cables for each 2-DoF section to minimise the number of actuators with a precise actuation. To be able to achieve the desired configuration shape, a kinematic model has been established and the configuration-cable kinematics has been implemented. Finally, the continuum robot has been built and tested for performing the predefined configuration shape.
\end{abstract}

\section{INTRODUCTION}

In-situ aeroengine repair can significantly reduce currently accepted maintenance cycle which is extensive and costly due to the need to remove engines from the wing of an aircraft to fully strip and overhaul. Over the past decade, robots have been integrated into many service operations around the world and have enabled or improved many new in-situ aeroengine repair technologies [1]. Currently, most employed devices are either rigid-segmented boreblending tools [2] with one DoF or flexible ones with two DoFs to perform bending movement in two directions [3]. However, the current designs of these devices are unable to cover a wide range of maintenance works as the limited DoFs and articulated lengths $(10-30 \mathrm{~mm})$, which prevent them to reach intervention places far away from the accessing ports.

Continuum robots, as a subset of hyper-redundant manipulators [4] which utilize compliant joints in series to create a highly flexible and compliant arm capable of intricate and

*Research supported by the Aerospace Technology Institute (UK) and Rolls-Royce Plc (Corresponding author: Xin Dong)

${ }^{1}$ Mingfeng Wang, David Palmer, Xin Dong, David Alatorre, Dragos Axinte are with the Rolls-Royce UTC in Manufacturing and On-Wing Technology, University of Nottingham, Nottingham, NG8 1BB, UK \{Mingfeng.Wang, David.Palmer, David.Alatorre, Xin.Dong, Dragos.Axinte\} enottingham.ac.uk

${ }^{2}$ Andy Norton is with Rolls-Royce plc, Derby, DE24 8BJ, UK Andy.NortoneRolls-Royce.com complex motions, have the potential to further advance the benefits of in-situ aeroengine repair and make new procedures possible. Typically the continuum robots are either used for grasping [5] or inspecting and performing actions in restrictive environments [6]. This paper focuses on the latter. Generally robots of this nature are designed to be allround performers, with every joint having the same reach and constraints. The reasoning behind this is to make the systems suitable for most scenarios not a singular case, which is true of [7] [8], and therefore separate themselves from purposebuilt mechanisms. There are some scenarios where this broad approach is not suitable, one such case is a torus shaped environment with confined entrances (e.g., aeroengine combustor chamber). In this situation, significantly different from the current designs of medical continuum robots $(5-12 \mathrm{~mm}$ in diameter but only 10-30 $\mathrm{mm}$ articulated lengths), the robot has to come with a slender design (i.e., low diameter-tolength ratio) and perform a singular tight bend and then continuous shallow curve.

To address these needs and challenges, this paper reports on a design and development of a slender (diameter-tolength ratio $<0.02$ ) dual-structure continuum robot that provides adequate number of DoFs (16) and high length (715 mm), which makes it able to feeding in/out of the constrained environments via confined inspection ports and then to perform a required (C-c) configuration shape for further repair operation purpose. In II, the mechanical design of the continuum robot is presented in terms of the technical requirements, conceptual design and corresponding main specifications. Then, a kinematic model is established based on a piecewise constant-curvature theory and corresponding configuration-cable kinematics is implemented in III. Finally, IV deals with the experimental setup for validating test of the built prototype and the characterization of the performance of the built continuum robot.

\section{Mechanical Design}

The technical requirements, conceptual design and corresponding main specifications of a slender dual-structure continuum robot are presented in this section, respectively. The continuum robot consists of two stages: body section and tip section, in which two different types of bevelled disks and backbones are adopted, respectively.

\section{A. Technical Requirements}

The design of the proposed slender continuum robot has been driven by the technical requirements for performing in-situ aeroengine repair via borescope inspection ports and 
the limitations for the proposed slender continuum robot are specified as follows:

- Limited inspection ports - the potentially useful borescope ports are limited in the combustor due to ancillary equipment attached to the engine casing;

- Minimum overall length sufficient to ensure all of the aeroengine combustor can be covered via limited inspection ports;

- Maximum diameter to allow access from all of the selected inspection ports of the aeroengine combustor;

- Maximum section length and minimum section bend angle to traverse inside of the combustor by following the constant-curvature middle line of the combustor;

- Minimum internal diameter appropriate to allow enough space in the central for accessing inspection and repair tools;

- Minimum payload of $0.25 \mathrm{~kg}$ at the tip to ensure appropriate end-effectors (e.g., inspection and repair tools) can be adopted.

\section{B. Conceptual Design}

To perform the requested in-situ repair tasks for specific aeroengines, a conceptual design of the slender dual-structure continuum robot is proposed, as shown in Fig 1.

To satisfy two main purposes (i.e., feeding in/out and repair) and reduce the complexity, a two-stage tendon-driven structure is adopted in the proposed conceptual design (see Fig 1(a)). In these two stages, the first stage, i.e., body, consists of ten 1-DoF sections and is able to feeding in/out with full coverage of entire aeroengine combustor via inspection ports, whilst the second stage, i.e., tip, consists of three 2DoF sections and is capable of performing 6-DoF movements for feeding in/out and repair, thus, the entire continuum robot has 16 DoFs allocated to 13 sections.

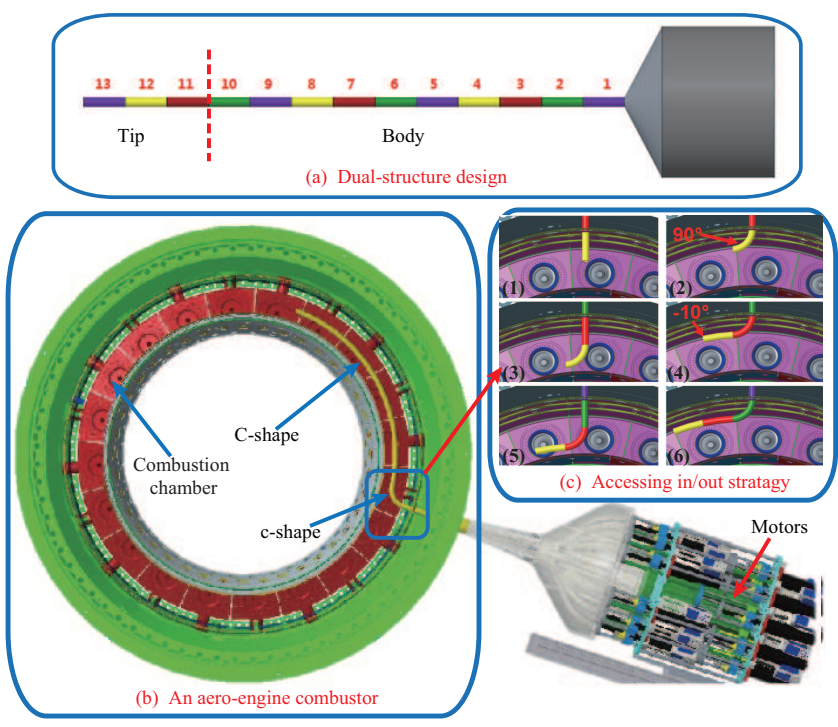

Fig. 1. A graphic representation of the conceptual design of the proposed dual-structure slender continuum robot: (a) a dual-structure design; (b)an aeroengine combustor; (c) a accessing in/out strategy.
A section-by-section strategy (see Fig. 1(c)) is used for feeding in/out the combustor since the section cannot start the bending movement before fully accessing into the combustor (the robot will collide with the edge of the panels) and correspondingly a C-c configuration shape of the continuum robot will be generated inside the combustor (see Fig. 1(b)) for further repair operations.

To achieve high payload capability at the tip and compensate the self-weight, a tendon-based and extrinsically actuated approach is applied in the continuum robot, where a pair of cables and a group of three cables are allocated in each body section and tip section, respectively. Furthermore, to prevent the backlash and slack, all cables are fully actuated which means 29 cable/motors (Maxon spindle drive: GP-32$\mathrm{S}-363904)$ in total are applied.

\section{Mechanical Specification}

The main specifications of mechanical design of the proposed dual-structure slender continuum robot are shown in 2 and listed in Table I.

A bevelled disk design with specific slopes is applied in the proposed continuum robot. In each disk of the body sections (see Fig. 2(a)), there are two bevels with the identical slope on one side and the slopes are different on two sides to perform a C-c bending shape, which is determined by the factors of the interval distance and the constant-curvature middle line of the combustor chamber. In each disk of the tip sections (see Fig. 2(b)), the slopes of four bevels on both sides are the same while two bevels on one side alternate at $90^{\circ}$ relative to the other side.

To achieve the light weight and enable the mechanical capabilities, the disks in the continuum robot are designed and manufactured by the titanium alloy because of its physical and mechanical properties such as light weigh, high tensile

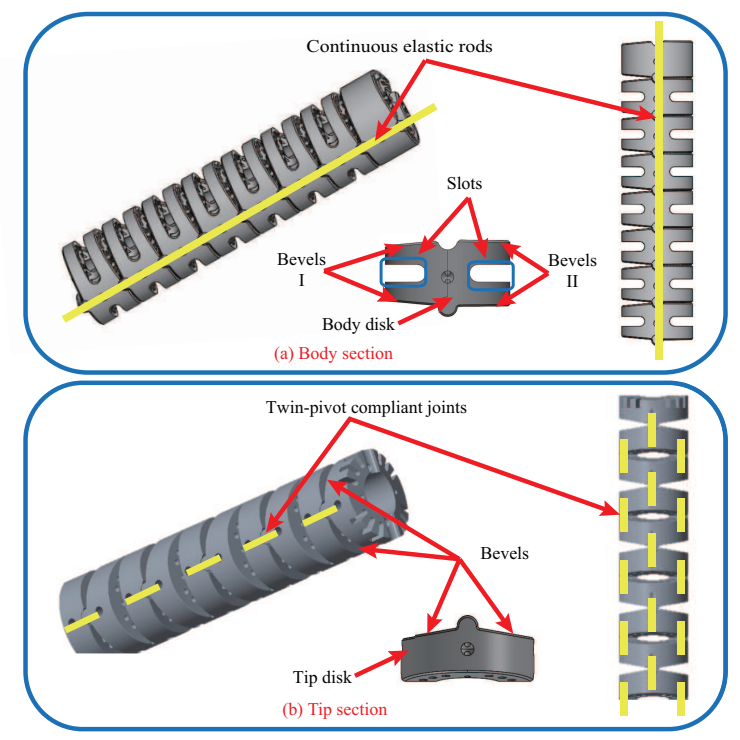

Fig. 2. A detailed representation of the main constitutive elements of the continuum robot: (a) continuous backbone and bevelled disk based design at the body sections; (b) twin-pivot compliant joint and bevelled disk based design at the tip sections. 
TABLE I

MAIN SPECIFICATION OF THE CONTINUUM ROBOT

\begin{tabular}{|c|c|c|}
\hline \multirow{2}{*}{ Continuum Robot } & \multicolumn{2}{|c|}{$\overline{\text { Stage }}$} \\
\hline & Body (10 sections) & Tip (3 sections) \\
\hline DoF & 10 (1 per section) & 6 (2 per section) \\
\hline Mass & $141.17 \mathrm{~g}$ & $51.99 \mathrm{~g}$ \\
\hline Length & $550 \mathrm{~mm}$ & $165 \mathrm{~mm}$ \\
\hline $\begin{array}{l}\text { Diameter-to-length } \\
\text { ratio }\end{array}$ & \multicolumn{2}{|c|}{$<0.02$} \\
\hline $\begin{array}{l}\text { Inner-to-outer } \\
\text { diameter ratio }\end{array}$ & \multicolumn{2}{|c|}{$>0.5$} \\
\hline Bending capability $^{a}$ & $-10^{\circ}$ to $+90^{\circ}$ & $-90^{\circ}$ to $+90^{\circ}$ \\
\hline Disk & 80 (8 per section) & 30 (10 per section) \\
\hline Backbone & $\begin{array}{l}\text { A pair of NiTi rods }(0.6 \\
\mathrm{mm})^{b}\end{array}$ & $\begin{array}{l}\text { A NiTi rod Twin- } \\
\text { pivot }(0.6 \mathrm{~mm})^{c}\end{array}$ \\
\hline Driven tendon & $\begin{array}{l}\text { Nylon covered stainless } \\
\text { steel wires }(0.90 \mathrm{~mm})\end{array}$ & $\begin{array}{l}\text { Nylon coverd steel } \\
\text { wire }(0.75 \mathrm{~mm})\end{array}$ \\
\hline
\end{tabular}

a. Bending capability in each section; b. A pair of NiTi rods run through all body sections; c. A NiTi rod twin-pivot is allocated between two disks (i.e.,one segment).

strength and toughness [9]. In each disk of body sections, two optimised slots have been placed to reduce the self-weight by almost $28 \%$ (see Fig. 2(a)).

In the body sections, a pair of continuous elastic rods (NiTi rods) is applied to enable the physical capabilities which motivate the continuum robot to adapt the backbone shape to conform the robot to the constant-curvature middle line of the combustor chamber (C-c shaped planar deformation). However, in the tip sections, to ensure the high flexibility and enhance torsional stability relative to the axis of the structure, a twin-pivot compliant joints (short NiTi rods) (alternating at $90^{\circ}$ to account for 2 DoFs in each section) structure [10] is adopted (see Fig. 2(b)).

\section{KinEMATICS ANALYSIS}

In this section, a piecewise constant-curvature theory based kinematic model is derived to form the basis for mechanical design, actuation specification and controller development.

\section{A. Kinematic Model}

The proposed continuum robot is divided into two stages (i.e., body and tip), as shown in Fig. 1, where the first stage (sections 1 to 10 ) can be modelled as a planar continuum manipulator and the second stage (sections 11 to 13) can be a spatial continuum manipulator. A tip-following algorithm based initial navigation strategy [11] is applied to generate the path for the entire robot and it allows the user to feed in the tip through a restrictive environment with the body following the tip's path. As aforementioned, in this case, only the tip sections are used for performing repair tasks whilst the body sections are formed with $\mathrm{C}$-c shape, which means the continuum robot is in an optimal position for operation with sections 1-10 fixed.

Based on piecewise constant-curvature theory, the continuum robot kinematics are generally decomposed into two submappings [12]. One is between joint or actuator space (e.g., length of cable, deformation of push rods/tubes, pressure of tube, etc.) and configuration space (i.e., arc

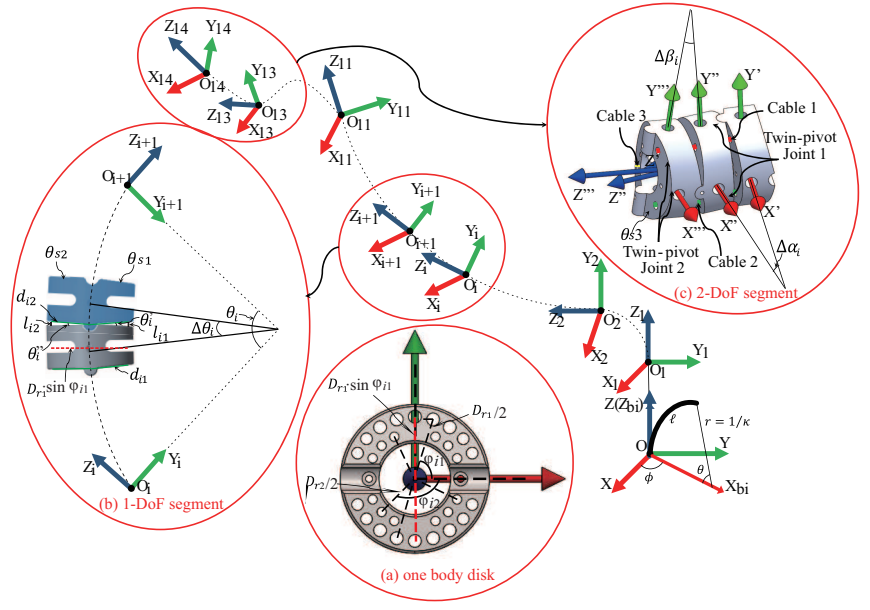

Fig. 3. Schematic for a kinematic model of the continuum robot: (a) top view of one disk (b) a single segment of the 1-DoF structure with continuous backbones in body sections; (c) a single segment of the 2-DoF structure with twin-pivot compliant joints in tip sections.

parameters in terms of the curvature $\kappa$, the rotational angle $\phi$ and the arc length $\ell$ ), while the other is between this configuration space and task space (i.e., position and pose of the end-effector). Furthermore, the former is a robotspecific mapping which means the kinematics varies with different actuation approaches (e.g., tendon-driven, flexible push rods/tubes, pneumatic tubes, etc.), while the latter is a robot-independent mapping which is general and applies to each independent actuated section.

To implement the kinematic analysis, the coordinate frames in terms of world frame, base disk frame $\left\{O_{i}\right\}(i=$ $1, \ldots, 13)$, end-effector (end disk of $13^{\text {th }}$ section) frame $\left\{O_{14}\right\}$, and bending frames $\left\{B_{i}\right\}$ are established (see Fig. 3 and Table II). Since tendon-driven is chosen as the actuation approach (i.e., the backbone arc is shaped by driving cables), the actuator space variables (i.e., the cable lengths) in $i^{\text {th }}$ section can be written in the forms of $L_{i}=\left[l_{i 1}, l_{i 2}, l_{i 3}\right]^{T}$, where $l_{i 3}$ equals to zero in sections 1-10 since only a pair of cables is utilized. The general kinematic representation of configuration space (arc parameters $q=[\kappa, \phi, \ell]^{T}$ ) is illustrated referring to world frame $\{O\}$ in Fig. 2, and the arc geometry relationships can be presented as

$$
\left\{\begin{array}{l}
\theta=\kappa \cdot \ell \\
r=1 / \kappa
\end{array}\right.
$$

where $\theta$ is the bending angle and $r$ is the bending radius. Specifically, the $+Z_{i}$-axes are considered to be always tangent to the constant-curvature curve and positive curvature $(\kappa>0)$ produces bending towards the $+\mathrm{X}_{b i}$-axes. Furthermore, the task space (i.e., the position and orientation of the end-effector) is represented by the position of the last disk centre $O_{14}=\left[x_{14}, y_{14}, z_{14}\right]^{T}$ and the rotation matrix of the frame $\left\{O_{14}\right\}:{ }^{0} R_{14} \in \mathfrak{R}^{3 \times 3}$.

\section{B. Configuration-Cable Kinematics}

As mentioned in II-A, a C-c shaped configuration is essential for the feed in/out and repair operation of the 
continuum robot. In this subsection, our scope is focused on the configuration-cable kinematics which provides the required input data for the experimental test of feed in/out with C-c shaped configuration in IV.

Once the aforementioned kinematic model is established, the purpose of configuration-cable kinematic analysis is to solve the mathematics to describe the lengths of cables $L=$ $\left[L_{1}, \cdots, L_{13}\right]^{T}$ relative to the given bending arc configuration in each section $q_{i}=\left[\kappa_{i}, \phi_{i}, \ell_{i}\right]^{T}$. Note that the cables are allocated to different holes in each disk with different pitch circle diameters (PCDs: $D_{r 1}$ and $D_{r 2}$ ) and phase angles $\varphi_{i}=\left[\varphi_{i 1}, \varphi_{i 2}, \varphi_{i 3}\right]^{T}$ (see Fig. 3(a)), the projection of each cable hole's PCD on bending plane can be expressed as



Furthermore, as each section includes identical segments and two different section structures are applied in body and tip respectively, the configuration-cable kinematics can be expressed by analysing two single segments in which one is from the body (Fig. 3(b)) and the other is from tip (Fig. 3(c)).

In Fig. 3(b), a single segment, which includes two adjacent disks and a continuous compliant joint, represents a basic 1DoF structure in $i^{\text {th }}$ section (body). Noting that the throughdisk cable lengths are constant, the contribution of arc parameters to the entire cable length only relates to the sum of changes of gap cable length $\Delta L_{i}$. According to the geometry relationships, the change of gap cable length can be expressed as (see Appendix for detail derivation)

$$
\left\{\begin{aligned}
\Delta l_{i 1} & =\frac{2 \cdot\left|D_{r 1} \cdot \sin \varphi_{i 1}\right| \cdot\left[\sin \left(\theta_{s 1}-\frac{\Delta \theta_{i}}{2}\right)-\sin \left(\theta_{s 1}\right)\right]}{\cos \theta_{s 1}} \\
\Delta l_{i 2} & =\frac{2 \cdot\left|D_{r 1} \cdot \sin \varphi_{i 2}\right| \cdot\left[\sin \left(\theta_{s 2}+\frac{\Delta \theta_{i}}{2}\right)-\sin \left(\theta_{s 2}\right)\right]}{\cos \theta_{s 2}}
\end{aligned}\right.
$$

where $\theta_{s 1}$ and $\theta_{s 2}$ are the slope angles of two planes, respectively and the $\Delta \theta_{i}$ is the segment bending angle with the bending angle $\theta_{i}$ which can be obtained by (1). Furthermore, the total change in length of the cable in the $i^{\text {th }}$ section (body) can be obtained by taking into account the disk number in each body section, $N_{\text {body }}$.

In Fig. 3(c), a single segment, which includes three adjacent disks and twin-pivot joints, represents a basic 2-DoF structure in the tip sections. A complete derivation of the

TABLE II

Nomenclature

\begin{tabular}{ll}
\hline \hline Symbol & Description \\
\hline$\{O\}: O-X Y Z$ & $\begin{array}{l}\text { World frame with origin, } O \text {, located at the } \\
\text { base }\end{array}$ \\
$\left\{O_{i}\right\}: O_{i}-X_{i} Y_{i} Z_{i}$ & $\begin{array}{l}\text { Base disk frame of } i^{\text {th }} \text { section }(i=1, \ldots, 13) \\
\text { with origin } O_{i} \text { at the centre }\end{array}$ \\
$\left\{B_{i}\right\}: O_{b i}-X_{b i} Y_{b i} Z_{b i}$ & $\begin{array}{l}\text { Bending plane frame of } i^{t h} \text { section }(i= \\
1, \ldots, 13), \text { where the } i^{t h} \text { section always bends } \\
\text { in the } y_{b i} z_{b i} \text { plane and the origin } O_{b i} \text { is }\end{array}$ \\
& $\begin{array}{l}\text { coincident with } O_{i} \\
\text { End disk frame of } 13^{\text {th }} \text { section with origin } \\
\left\{O_{14}\right\}: O_{14}-x_{14} y_{14} z_{14} \\
O_{14} \text { at the centre }\end{array}$ \\
\hline \hline
\end{tabular}

inverse kinematics of a twin-pivot compliant joints structure is given in [10]. However, due to four in two pairs of cables allocated to ordinary disks are used to shape the arc in [10] while a group of three cables through the bevelled disks are used in this case, the kinematics of the single segment needs to be discussed in detail. Referring to [10], the bending angles of twin-pivot joints 1 and 2 (i.e., $\Delta \alpha_{i}$ and $\Delta \beta_{i}$ ) in single segment can be expressed with respect to the bending and direction angles of the corresponding section as written by Eq. (18) in [10]. Based on the obtained angle values of $\Delta \alpha_{i}$ and $\Delta \beta_{i}$ and similar geometry relationships in body segment, the change of cable lengths in gap 1 (with bending angle of $\Delta \alpha_{i}$ ) can be expressed as

$$
\left\{\begin{aligned}
\Delta l^{\prime}{ }_{i 1} & =\frac{2 \cdot\left|D_{r 2} \cdot \sin \varphi_{i 1}\right| \cdot\left[\sin \left(\theta_{s 3}-\frac{\Delta \alpha_{i}}{2}\right)-\sin \left(\theta_{s 3}\right)\right]}{\cos \theta_{s 3}} \\
\Delta l^{\prime}{ }_{i 2} & =\frac{2 \cdot\left|D_{r 2} \cdot \sin \varphi_{i 2}\right| \cdot\left[\sin \left(\theta_{s 3}-\frac{\Delta \alpha_{i}}{2}\right)-\sin \left(\theta_{s 3}\right)\right]}{\cos \theta_{s 3}} \\
\Delta l^{\prime}{ }_{i 3} & =\frac{2 \cdot\left|D_{r 2} \cdot \sin \varphi_{i 3}\right| \cdot\left[\sin \left(\theta_{s 3}+\frac{\Delta \alpha_{i}}{2}\right)-\sin \left(\theta_{s 3}\right)\right]}{\cos \theta_{s 3}}
\end{aligned}\right.
$$

Similarly, the change of cable lengths in gap 2 (with bending angle of $\Delta \beta_{i}$ ) can be written as

$$
\left\{\begin{aligned}
\Delta l^{\prime \prime}{ }_{i 1} & =\frac{2 \cdot\left|D_{r 2} \cdot \cos \varphi_{i 1}\right| \cdot\left[\sin \left(\theta_{s 3}-\frac{\Delta \beta_{i}}{2}\right)-\sin \left(\theta_{s 3}\right)\right]}{\cos \theta_{s 3}} \\
\Delta l^{\prime \prime}{ }_{i 2} & =\frac{2 \cdot\left|D_{r 2} \cdot \cos \varphi_{i 2}\right| \cdot\left[\sin \left(\theta_{s 3}+\frac{\Delta \beta_{i}}{2}\right)-\sin \left(\theta_{s 3}\right)\right]}{\cos \theta_{s 3}} \\
\Delta l^{\prime \prime}{ }_{i 3} & =\frac{2 \cdot\left|D_{r 2} \cdot \cos \varphi_{i 3}\right| \cdot\left[\sin \left(\theta_{s 3}+\frac{\Delta \beta_{i}}{2}\right)-\sin \left(\theta_{s 3}\right)\right]}{\cos \theta_{s 3}}
\end{aligned}\right.
$$

Therefore, according to 4 and 5, the total change in length of the cable in the $i^{\text {th }}$ section (tip) can be also obtained by taking into account the disk number in each tip section, $N_{t i p}$.

\section{EXPERIMENTAL VALIDATION}

With the mechanical design and the key kinematic modelling commented, a prototype of the proposed continuum robot has been built as shown in Fig.4. The validation of the capabilities of this complex mechatronic system has been performed to check some key characteristics to the targeted demonstration in this section.

\section{A. An Experimental Layout}

To validate the design and development of the continuum robot it is required to perform the $\mathrm{C}$-c configuration shape at the specified curvature and centre. An experimental layout of the built prototype is presented, as shown in Fig. 4, where a VICON optical motion capture system is used to measure the movement with a standard setup for capturing data. Markers are added on small square plates which are mounted on the base of continuum robot arm and the end disks of each section, allowing the tracking of the position and orientation of each marker plate (i.e., represent the base disk frames $\left\{O_{i}\right\}$ and end-effector frame $\left\{O_{14}\right\}$ ). The central point of the curve can be determined from where the normals of each marker plate intersect, while the curvature of each section can be calculated by the relation between neighbouring markers. In addition, a low-level control system has been developed using LabVIEW FPGA and linear encoders have been integrated with the actuation pack of each motor, allowing the close-loop control of cable lengths. 


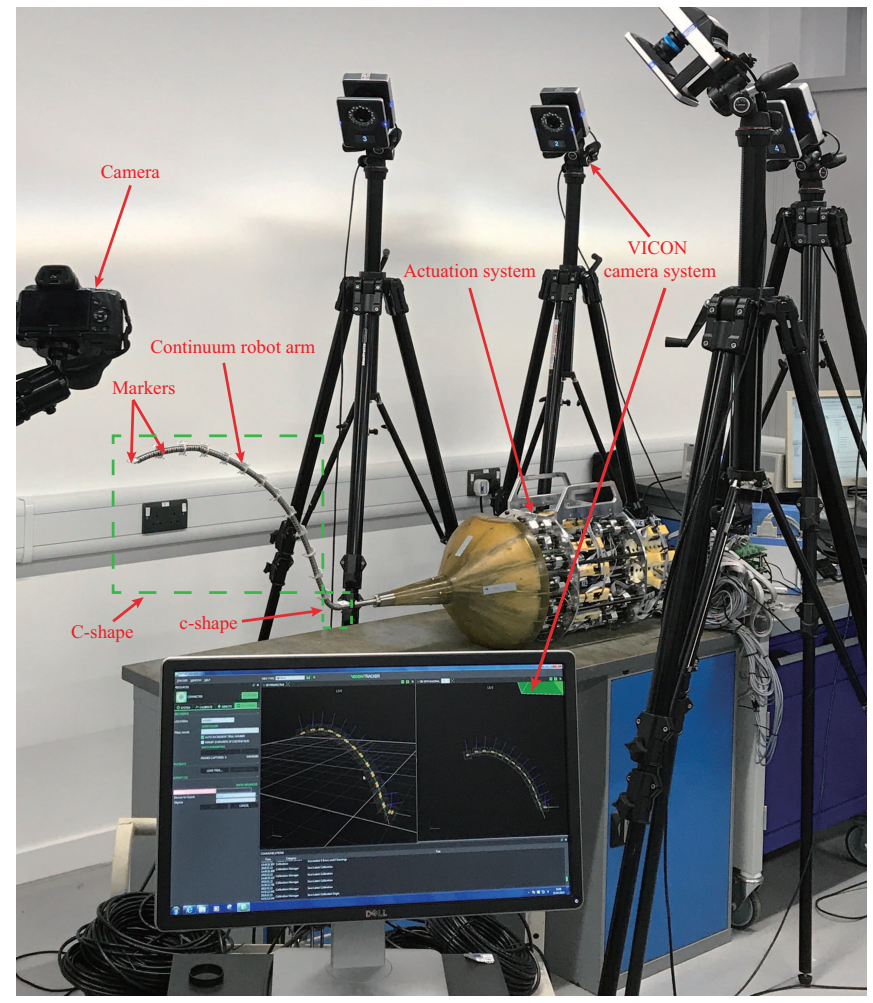

Fig. 4. An experimental layout of the continuum robot with VICON camera system.

\section{B. Experimental Test Results}

The accuracy of the system is dependent on the precision of the cable actuation, especially as the behaviour of the backbones in the continuum robot can be considered as an open-loop system. Fig.5 compares the desired change in the cable length (solid lines) against the actual (dashed lines) for two sets of cables (i.e., one pair of cables in one body section and a group of three cables in one tip section) in the system while it is performing the $\mathrm{C}$-c shape movement with increasing number of sections. The maximum $\Delta l$ in the test was $7.12 \mathrm{~mm}$ which produced the maximum error of 0.66 $\mathrm{mm}$ (9.3\% of the desired). The average error was measured to be less than the $2.5 \mu \mathrm{m}$ step size of the linear encoder, and therefore the minimal achievable error.

From this experiment it is possible to compare the actuated position against the simulated position of the system. The graph depicted in Fig.6 represents the continuum robot in two poses: the initial position and the full C-c configuration shape. On both poses, the simulated robot is represented as a solid line whilst the VICON data is a dashed line connecting the markers.

There is a notable undershoot from the system. It can be characterised using two methods, by the resulting radius of the curve and the bending angle of the curve. The central point of the curve can be determined from where the normals of each marker plate intersect, while the curvature of each section can be calculated by the relation between neighbouring markers. Furthermore, in Fig. 6, the errors of

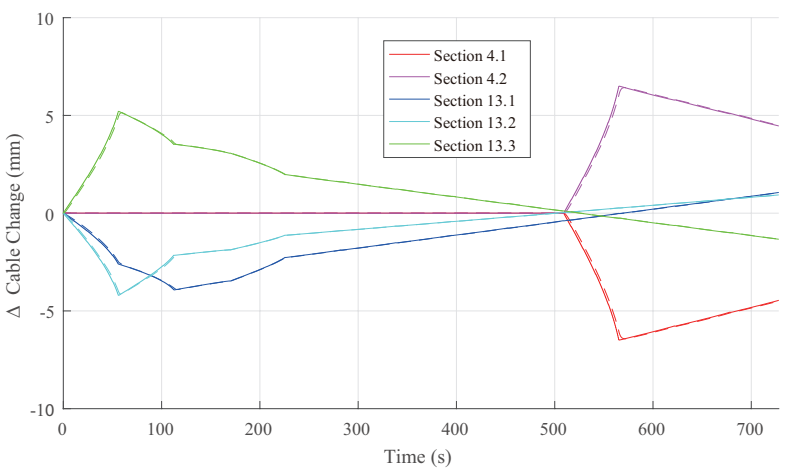

Fig. 5. Cable length changes during performing C-c configuration shape (section 4 in body and section 13 in tip were selected as an example to represent).

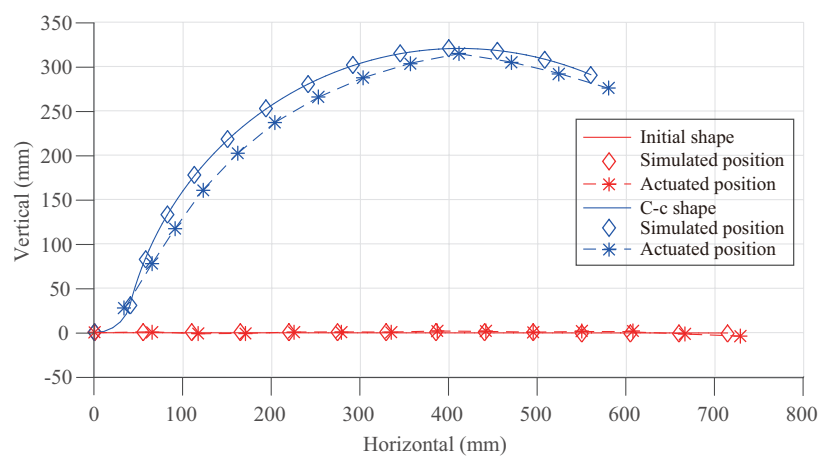

Fig. 6. A bending test to perform C-c configuration shape.

radius and curvature between the simulated and actuated positions can be calculated and they are less than $10 \%$, which are theorized to be caused either by slack in the cables or the elastic stretch when under tension. Whilst the tests results in this paper demonstrate that the system is sufficient to meet the requirements in II-A, further work will be focus on improving this performance (e.g., the uneven bend in section 11) by pre-calibration based on the geometry limits of the bevels in disks and force compensation of the cables.

\section{CONCLUSIONS}

This paper presents on a the route to design and develop a highly slender (diameter-to-length $<0.02$ ) dual-structure continuum robot (16 DoFs) to be utilised on inspection and repair of aeroengine combustor via borescope ports. The mechanical design relies on a two-stage structure with 13 sections (ten 1-DoF body sections and three 2-DoF tip sections) based on customised bevelled disks and compliant joints. Kinematic models have been developed and configuration-cable kinematics has been solved to enable the control of the multi-section robot reaching the desired C-c configuration shape for feed in/out the combustor and further repair operation. Finally, the continuum robot has been successfully built and tested by performing a C-c 
configuration shape bending with max. error of $0.66 \mathrm{~mm}$ in actuating the cable length of $7.12 \mathrm{~mm}$ and max. deviations of the curve is less than $10 \%$. Thus, this paper presents a slender continuum robot that could be considered as a step forward in providing aeroengine manufacturers with a solution to perform complex tasks.

\section{APPENDIX}

As shown in Fig. 3(a) and (b), the distance between the centre of cable hole and Z-axis can be expressed as

$$
\left\{\begin{array}{l}
d_{i 1}=\left|D_{r 1} \cdot \sin \varphi_{i 1}\right| \cdot \sin \left(\theta_{s 1}\right) \\
d_{i 2}=\left|D_{r 1} \cdot \sin \varphi_{i 2}\right| \cdot \sin \left(\theta_{s 2}\right)
\end{array}\right.
$$

and the angles between the two inclined planes with slope angles $\theta_{s 1}$ and $\theta_{s 2}$ can be written as

$$
\left\{\begin{array}{c}
\theta_{i}^{\prime}=2 \theta_{s 1}-\Delta \theta_{i} \\
\theta^{\prime \prime}{ }_{i}=2 \theta_{s 2}+\Delta \theta_{i}
\end{array}\right.
$$

where the bending angle $\Delta \theta_{i}$ can be calculated according to the arc configuration and (1). Thus, the change of gap cable length $\Delta L_{i}=\left[\Delta l_{i 1}, \Delta l_{i 2}, 0\right]^{T}$ can be obtained as

$$
\left\{\begin{array}{l}
\Delta l_{i 1}=\sqrt{2 \cdot d_{i 1}{ }^{2}\left(1-\cos \theta_{i}^{\prime}\right)}-\sqrt{2 \cdot d_{i 1}{ }^{2}\left(1-\cos 2 \theta_{s 1}\right.} \\
\Delta l_{i 2}=\sqrt{2 \cdot d_{i 2}{ }^{2}\left(1-\cos \theta^{\prime \prime}{ }_{i}\right)}-\sqrt{2 \cdot d_{i 2}{ }^{2}\left(1-\cos 2 \theta_{s 2}\right)}
\end{array}\right.
$$

Therefore, according to A.1 to A.3, 5 can be derived.

\section{ACKNOWLEDGMENT}

The research leading to these results has received funding from the Aerospace Technology Institute (UK) under Grant Agreement No. 102360 (FLARE) and Rolls-Royce Plc.

\section{REFERENCES}

[1] X. Dong, D. Axinte, D. Palmer, S. Cobos, M. Raffles, A. Rabani, and J. Kell, "Development of a slender continuum robotic system for on-wing inspection/repair of gas turbine engines," Robotics and Computer-Integrated Manufacturing, vol. 44, no. April, pp. 218-229, 2017.

[2] H. Heckele, F. Hähnle, and E. Körner, "Surgical instrument for applying implants," Patent US 6830 574B2, 2004.

[3] J. Diener, "Instrument for working the surfaces of parts inside engineered cavities," Patent US 5475 485A, 1995.

[4] G. Chirikjian and J. Burdick, "Hyper-redundant robot mechanisms and their applications," Proceedings IROS '91:IEEE/RSJ International Workshop on Intelligent Robots and Systems '91, no. 91, pp. 185-190, 1991.

[5] W. McMahan, V. Chitrakaran, M. Csencsits, D. Dawson, I. D. Walker, B. Jones, M. Pritts, D. Dienno, M. Grissom, and C. D. Rahn, "Field trials and testing of the OcotArm continuum manipulator," Proceedings of the 2006 IEEE International Conference on Robotics and Automation (ICRA), pp. 2336-2341, 2006.

[6] R. Buckingham, "Snake arm robots for flexible delivery," Insight: NonDestructive Testing and Condition Monitoring, vol. 44, no. 3, pp. 150151, 2002.

[7] I. D. Walker, "Continuous Backbone Continuum Robot Manipulators," ISRN Robotics, vol. 2013, pp. 1-19, 2013.

[8] X. Dong, M. Raffles, S. C. Guzman, D. Axinte, and J. Kell, "Design and analysis of a family of snake arm robots connected by compliant joints," Mechanism and Machine Theory, vol. 77, pp. 73-91, 2014.

[9] R. Jing, S. Liang, C. Liu, M. Ma, X. Zhang, and R. Liu, "Structure and mechanical properties of Ti6A14V alloy after zirconium addition," Materials Science and Engineering: A, vol. 552, pp. 295-300, aug 2012.
[10] X. Dong, M. Raffles, S. Cobos-Guzman, D. Axinte, and J. Kell, "A Novel Continuum Robot Using Twin-Pivot Compliant Joints: Design, Modeling, and Validation," Journal of Mechanisms and Robotics, vol. 8, no. 2, p. 21010, 2015.

[11] D. Palmer, S. Cobos-Guzman, and D. Axinte, "Real-time method for tip following navigation of continuum snake arm robots," Robotics and Autonomous Systems, vol. 62, no. 10, pp. 1478-1485, 2014.

[12] R. J. Webster and B. A. Jones, "Design and Kinematic Modeling of Constant Curvature Continuum Robots: A Review," The International Journal of Robotics Research, vol. 29, no. 13, pp. 1661-1683, 2010. 